\title{
Reed-Solomon Code Performance for GMSK Modulation over AWGN Channel
}

\author{
${ }^{*}$ Monika Kapoor ${ }^{1}$, Dr.AnubhutiKhare ${ }^{2}$ \\ ${ }^{I}$ Ph.D Scholar Electronics \& Communication Engg.UIT RGPVBhopal,India \\ ${ }^{2}$ Electronics \& Communication Engg.UIT Rajiv Gandhi ProuduogikiVishwavidalayaBhopal,India \\ Corresponding Author: Monika Kapoor
}

\begin{abstract}
The main objective of this paper is to find the performance of Reed-Solomon code for Guassian Minimum Shift Keying (GMSK) modulation overa Additive White Guassian Noise (AWGN) channel. The simulation model provides error detection and correction using Reed-Solomon (RS) Codes. The Original message is encoded and decoded using RSCodes.MATLAB simulation is selected as the investigating tool.Theperformance of proposed GMSK and RS code combination simulation is compared with uncoded system with the constraint that the transmission bandwidth is constant. Wealsoperformed the simulations for different code rates and different block lengths with fixed number of error. The results are presented by a plot between the bit errorrates (BER) and signal to noise ratio using Monte Carlo simulation.Theresultsshowthatforagiven bandwidth, it is beneficial to use a smaller coderate but only to a certain value otherwise the system performance deteoriates. The BERperformance also improves by decreasing code rate and by taking large block lengths or by increasing redundancy.
\end{abstract}

Indexterms: $\quad A W G N, \quad B E R$ (Bit Error Rate),GMSK,Matlab,RSCodes,Partial Band Noise jamming (PBNJ), Galois field.

\section{Introduction}

To achieve consistent and reliable data from the information source to the destination is one of the mainissueincommunicationsystem. Themainobjective of any communication system is transmission of data with the minimum error rate no matter whether it is digital or analog. The use of channel codes or Forward Error Correcting Codes in digital communication system is an integral part of ensuring reliable communication[1] even in the presence of jamming.Jamming is an active attack with a purpose to prevent devices from exchanging information by interfering with their communication. There are various ways to counter jamming effect like making use of highly directional Antennas,using Forward Error Correcting Codes \& Spread Spectrum Communication.In case of Partial Band Noise jamming(PBNJ) jammer evenly distributes noise power over some frequency bandwidth which is a subset total bandwidth. So as to mitigate this PBNJ effect one Forward Error Correcting Code is required which is more suitable to work against Burst noise.Although there are various codes which works efficiently good for random errors but for Burst noise or Burst Error Reed Solomon Code is the best.

Reed Solomon are non binary byte organised codes which is widely used in wireless communication,compactdiscplayers and computers memories.Reed Solomon Codes are effective for deep fade channel and are considered as a structured sequence that is most widely used in Burst Error Control.

The main objective of this paper is to evaluate the performanceof Reed-Solomon codes in error correction controlsystemin term of bit error rate (BER). In proposedcommunication system the signal is transmitted using GMSK modulation technique in the presence of Additive White Gaussian Noise (AWGN). In GMSK, which is a subclass of continous phase modulation the digital data sream is first shaped with Gaussian filter before being applied to MSK modulator.By using Guassianfilter,sideband power get reduced which in turn yields excellent performance in the presence of Interchannel interference (ICI). Compressing the bandwidth although avoids ICI but it causes an expansion in time domain which results in IntersymbolInterference(ISI). In order to remove ISI effect Equalizers are required at the receiver end.

GMSK modulation method, first proposed by K.Murota and K.Hirade [4], is a widely used modulaion scheme of cellular system due to its compact Power Spectral Density and excellent error performance. Although the performance of GMSK has been analyzed by several researchers, coding for GMSK has received little attention[5].

This paper focuses on GMSK and Reed-Solomon (RS) coding. Error control codes insert redundancy into the transmitted data stream so that the receiver can correct errors that occur during transmission. Therefore, the bit interval of the coded bits is selected shorter in order to keep the information transmission rate constant. A 
shorter bit interval results in a larger transmission bandwidth. To remain the bandwidth of the coded system same as that of the uncoded system, the modulator used in the coded system must adopt a smaller value of $\mathrm{B}_{\mathrm{b}}[3]$.

This paper is organized as follows. The next section i.e. section 2 gives an overview of the system including a description of GMSK modulation and RS codes. Section 3 gives our approach to the bandwidth allocation problem. Simulation results for GMSK modulation with different Reed-Solomon coding rates for maintaining same bandwidth are presented in Section 4, and the conclusions are given in Section 5.

\section{System Overview}

RS/GMSK system model and Reed-Solomon coding system shown in Figure 1. The performance of various combinations of GMSK and RS codes is evaluated with the constraint that the total system bandwidth is constant. The bandwidth of GMSK canbe easily controlled by the parameter $\mathrm{B}_{\mathrm{b}}$. The uncoded system is also evaluated to serve as a benchmark.

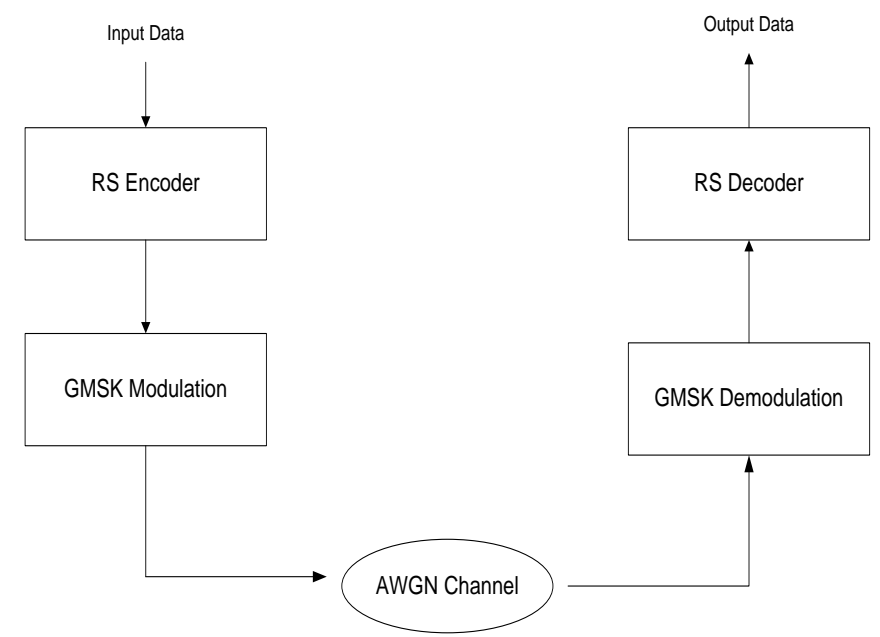

\section{A. GMSK Modulation}

Fig. 1. RS/GMSK Model

GMSK, as its name suggests, is based on MSK and was developed to improve the spectral properties ofMSK by using a premodulation Gaussian filter. The filter impulse response is expressed as:

$$
\begin{aligned}
& \boldsymbol{h}(\boldsymbol{t})=\frac{1}{\sqrt{2 \pi \sigma T}} \exp \left(\frac{-t^{2}}{2 \sigma^{2} T^{2}}\right) \ldots \ldots(1) \\
& \text { where } \sigma=\frac{\sqrt{\ln 2}}{2 \pi B_{b} T}
\end{aligned}
$$

The Gaussian filter is characterized by its $B_{b} T$ product $\left(B_{b}\right.$ is the $-3 d B$ bandwidth of the Gaussian prefilter and $\mathrm{T}$ is the symbol period.) The lower the $\mathrm{B}_{\mathrm{b}} \mathrm{T}$ product, the narrower the modulation bandwidth. In this paper, we use $B_{b} T=1.0$ and $B_{b} T=0.5$ for the uncoded system. For transmission in an AWGN channel, the bit error rate of GMSK is given by

$P=\frac{1}{h^{2}} \operatorname{erfc}\left(\sqrt{\frac{d_{\min }^{2} E}{2 N_{0}}}\right)$

Whered $_{\min }$ is the normalized minimum Euclidean distance between the signal representing " 0 " and the signal representing " 1 ", $E$ is the energy per transmitted bit and $\mathrm{N}_{0} / 2$ is the power spectral density of the AWGN.

\section{B. Reed-Solomon codes}

Reed-Solomon codes are block-based error correcting codes with a wide range of applications in digital communications and storage. It is vulnerable to the random errors but strong to burst errors. Hence, it has good performance in fading channel which have more burst errors. In coding theory Reed-Solomon (RS) codes are cyclic error correcting codes invented by Irving S.Reed and Gustave Solomon [6]. They described a systematic way of building codes that could detect and correct multiple random symbol errors. By adding $t$ check symbols to the data, an RS code can detect any combination of up to $t$ erroneous symbols, and correct up to [t/2] symbols. As an erasure code, it can correct up to $t$ known erasures, or it can detect and correct combinations of errors and erasures. Reed-Solomon codes are used to correct errors in many systems including:

- Storage devices (including tape, Compact Disk, DVD, barcodes, etc)

- Wireless or mobile communications (including cellular telephones, microwave links, etc) 
- Satellite communications

- Digital television / DVB

- High-speed modems such as ADSL, xDSL, etc.

Block coding schemes involve dividing the input data into k-bit blocks and then mapping each kbit block into an n-bit block called a codeword, where $n>k$ in the encoding process. (n-k) check bits are added to each $\mathrm{k}$-bit block. The ratio $\mathrm{r}=\mathrm{k} / \mathrm{n}$ is called the code rate. The data is partitioned into symbols of $\mathrm{m}$ bits, and each symbol is processed as one unit both by the encoder and decoder. RS codes satisfy: $n \leq 2^{m-1}$ and $n-k \geq 2 t$, where $t$ is the number of correctable symbol errors. Reed Solomon codes are polynomial codes over certain finite fields particularly useful in Burst error correction. Encoding \& Decoding principles of nonbinary RS codes depends on Galois fields (GF).Symbols from extension galois field $\left(2^{\mathrm{n}}\right)$ are used in constructing RS codes. GF $\left(2^{\mathrm{n}}\right)$ is extension galois field with $2^{n}$ elements.Let $\beta$ be a primitive element inGF $\left(2^{n}\right) \& G(Z)$ be the Generator polynomial with roots $\left(\beta, \beta^{2}, \beta^{3}, \ldots \ldots \ldots \beta^{\mathrm{N}-\mathrm{M}}\right)$. Then

$$
G(Z)=\sum_{I=1}^{N-M}\left(Z-\beta^{i}\right)
$$

Let $\left(\mathrm{m}_{1}, \mathrm{~m}_{2}, \ldots \ldots \mathrm{m}_{\mathrm{M}}\right)$ be the message symbols where $\mathrm{m}_{\mathrm{i}} \mathrm{EGF}\left(2^{\mathrm{n}}\right)$ which is defined by a polynomial $P(Z)=m_{1}+m_{2} Z+m_{3} Z^{2}+\ldots+m_{M} Z^{M-1}$

$\&$ HenceCodeword Polynomial is

$C(Z)=P(Z) G(Z) \ldots \ldots . .(5)$ If during transmission some additive errors are introduced due to noise which is described by error polynomiale $(Z)=\sum_{j=0}^{n-1} e_{i} Z^{i}$ then received polynomial becomes $R(Z)=C(Z)+$ $e(Z) \ldots . . .(7)$

Various algebric Decoding methods like Peterson-Gorenstein-Zierler(PGZ),Berlekamp- Massey Algorithm(BMA) and Euclidean method of Sugiyama are used for RS codes which are based on the idea of determining error location and error correction.Decodingalgorithm for t error correcting RS codes is based on considering errorpolynomiale $(\mathrm{Z})$ which is

$e(Z)=e_{n-1} Z^{n-1}+e_{n-2} Z^{n-2}+\ldots e_{1} Z+e_{0}$

here $\mathrm{v}$ is total errors that actually occurs \& $\mathrm{t}$ is error correcting capability of RS codes.Let these errors occur at locations $i_{1}, i_{2}, i_{3}, \ldots i_{v}$. The error polynomial can then be written as

$e(Z)=e_{i_{1}} Z^{i_{1}}+e_{i_{2}} Z^{i_{2}}+\cdots e_{i_{v}} Z^{i^{v}}$

Let The RS decoded symbol error probability, $\mathrm{P}_{\mathrm{E}}$, in terms of the channel symbol error probability, $\mathrm{p}$, can be written as follows [7]:

$P_{e} \approx \frac{1}{2^{m}-1} \sum_{j=t+1}^{2^{m}-1} j\left(\begin{array}{c}2^{m}-1 \\ j\end{array}\right) p^{j}(1-p)^{2^{m}-1-j}(5)$

Since RS code parameters have a significant effect on coding performance, RS codes with different rates are teste here $e_{i_{k}}$ is the magnitude of $\mathrm{k}^{\text {th }}$ error.For error correction we must know two things error locations $\&$ magnitude of these errors. Thus, the unknowns are $i_{1}, i_{2}, i_{3}, \ldots i_{v} \&, e_{i_{1}} e_{i_{2}}, . e_{i_{v}}$, which signify the locations \& the magnitudes of the errors respectively. The syndrome can be obtained by evaluating the received polynomial at $\alpha$

$$
S_{1}=v(\alpha)=c(\alpha)+e(\alpha)=e(\alpha)=e_{i_{1}} Z^{i_{1}}+e_{i_{2}} Z^{i_{2}}+\cdots e_{i_{v}} Z^{i_{v}}
$$

If error magnitudes are defined as $Y_{k=} e_{i_{k}}$ for $k=1,2, \ldots v \&$ error locations are $Z_{k=} \propto^{i_{k}} \quad$ for $k=1,2, \ldots v$, where $\mathrm{i}$ ${ }_{\mathrm{k}}$ is the location of $\mathrm{k}^{\text {th }}$ error $\& \mathrm{Z}_{\mathrm{k}}$ is the field element associated with this location then Syndrome can be written as $S_{1}=Y_{1} Z_{1}+Y_{2} Z_{2}+\ldots Y_{v} Z_{v}$

We can evaluate the received polynomial at each of the powers of $\alpha$, thus we have following set of $2 t$ equations with $\mathrm{v}$ unknown error locations $Z_{1}, Z_{2}, \ldots, Z_{v}$ \& the $v$ unknown error magnitudes $Y_{1}, Y_{2}, \ldots, Y_{v}$,

$S_{1=} Y_{1} Z_{1}+Y_{2} Z_{2}+\cdots+Y_{v} Z_{V}$

$S_{2}=Y_{1} Z_{1}^{2}+Y_{2} Z_{2}^{2}+\cdots+Y_{v} Z_{v}^{2}$

$S_{2 t}=Y_{1} Z_{1}^{2 t}+Y_{2} Z_{2}^{2 t}+\cdots Y_{v} Z_{v}^{2 t}$

If the error locator polynomial is defined as

$A(z)=A_{v} z^{v}+A_{v-1} z^{v-1}+\cdots A_{1} z+1$

Then zeros of this polynomial are the inverse error locations $Z_{k}^{-1}$ for $k=1,2, \ldots, v$ i.e.

$A(z)=\left(1-z Z_{1}\right)\left(1-z Z_{2}\right) \ldots\left(1-z Z_{V}\right) \ldots . .(16)$

So,if we know the coefficients of the error locator polynomial $A(z)$, we can obtain the error locations $\mathrm{Z}_{1}, \mathrm{Z}_{2}, \ldots . \mathrm{Z}_{\mathrm{v}}$. Since error locations are now known these form a set of $2 \mathrm{t}$ linear equations. These can be solved to obtain error magnitudes. 


\section{III.PERFORMANCE EVALUATION}

In order to determine what combination of coding rate and $\mathrm{B}_{\mathrm{b}}$ for the coded system results in the same bandwidth as the uncoded system, we must choose the measure of bandwidth. In this paper, we have used the percent power containment bandwidth, denoted by $\mathrm{B}_{\mathrm{x}}$ and defined as the bandwidth which contains $\mathrm{x} \%$ of the signal power. $\mathrm{B}_{90}, \mathrm{~B}_{99}$ and $\mathrm{B}_{99: 9}$ are plotted in Figure 2 .

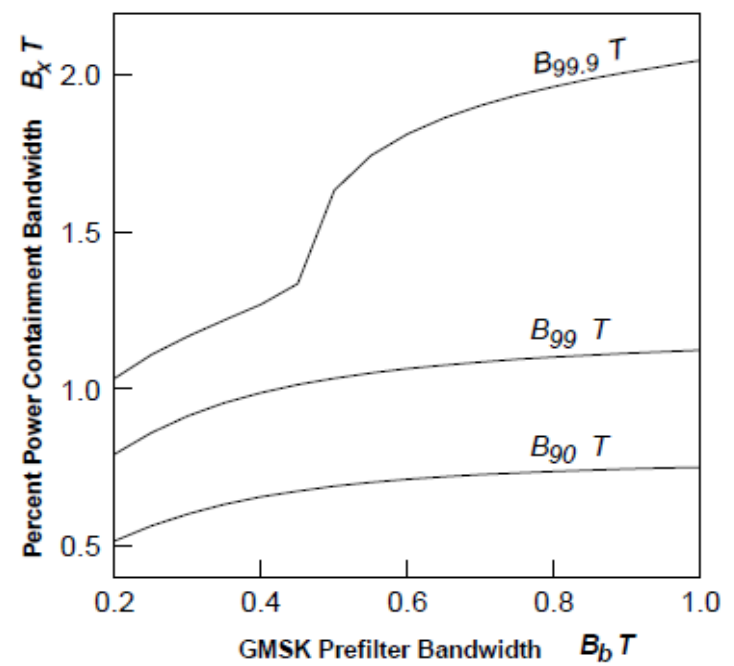

Fig. 2. Percent power containment bandwidths for GMSK

$\mathrm{B}_{99: 9}$ for GMSK, which is the bandwidth that contains $99: 9 \%$ of the signal power is used in the simulations. In Equation (2) for the uncoded system, the value of $\mathrm{E}$ is $\mathrm{E}_{\mathrm{b}}$, which is the energy per transmitted information bit. For the coded system, the value of $\mathrm{E}$ is set to be $\mathrm{rE}_{\mathrm{b}}$, since the energy for the coded bits is spread among the more numerous coded bits. This allows a fair comparison to be made between the uncoded and coded systems.It is complicated to compute the bit error probability $\mathrm{p}_{\mathrm{b}}$ by using Equation (2) and (3) because RS codes are nonbinary codes, so we use MATLAB. The simulation model is shown in Figure 3.

In the simulations the following parameters are used.

- Input data 100000 symbols

- $\quad$ RS codeword length: $31,63,127$

SIMULATION PARAMETERS

\begin{tabular}{|c|c|}
\hline Item & Value \\
\hline Channel Model & AWGN \\
\hline Modulation & GMSK \\
\hline Channel Coding & Reed Solomon \\
\hline Codeword Length & $127,63,31$ \\
\hline Data Rate & $1 \mathrm{Mbps}$ \\
\hline Frequency & $850 \mathrm{MHz}$ \\
\hline
\end{tabular}

$B_{\mathrm{B}}$ TAND RS CODE PARAMETER COMBINATIONS THAT RESULT IN SYSTEMS WITH EQUAL BANDWIDTHS

\begin{tabular}{|c|c|c|c|}
\hline \multirow{4}{|c|}{$\mathbf{n = 3 1}$} \\
\hline \multirow{2}{*}{ Uncoded } & $\mathbf{3}$ Coded \\
\cline { 2 - 4 } & $\mathbf{k = 2 5}$ & $\mathbf{k = 2 3}$ & $\mathbf{k = 2 1}$ \\
\hline 0.5 & 0.45 & 0.35 & 0.25 \\
\hline 1.0 & 0.51 & 0.48 & 0.45 \\
\hline
\end{tabular}

\begin{tabular}{|c|c|c|c|}
\hline \multicolumn{4}{|c|}{$\mathbf{n = 6 3}$} \\
\hline \multirow{2}{*}{ Uncoded } & \multicolumn{3}{|c|}{ Coded } \\
\cline { 2 - 4 } & $\mathbf{k}=\mathbf{5 7}$ & $\mathbf{k = 5 3}$ & $\mathbf{k = 4 9}$ \\
\hline 0.5 & 0.47 & 0.46 & 0.40 \\
\hline 1.0 & 0.64 & 0.54 & 0.49 \\
\hline
\end{tabular}

\begin{tabular}{|c|c|c|c|}
\hline \multirow{4}{*}{$\mathbf{n}=\mathbf{1 2 7}$} \\
\hline \multirow{3}{*}{ Uncoded } & \multicolumn{3}{|c|}{ Coded } \\
\cline { 2 - 4 } & $\mathbf{k}=\mathbf{1 0 7}$ & $\mathbf{k = 1 0 3}$ & $\mathbf{k = 9 9}$ \\
\hline 0.5 & 0.47 & 0.45 & 0.40 \\
\hline 1.0 & 0.54 & 0.51 & 0.49 \\
\hline
\end{tabular}




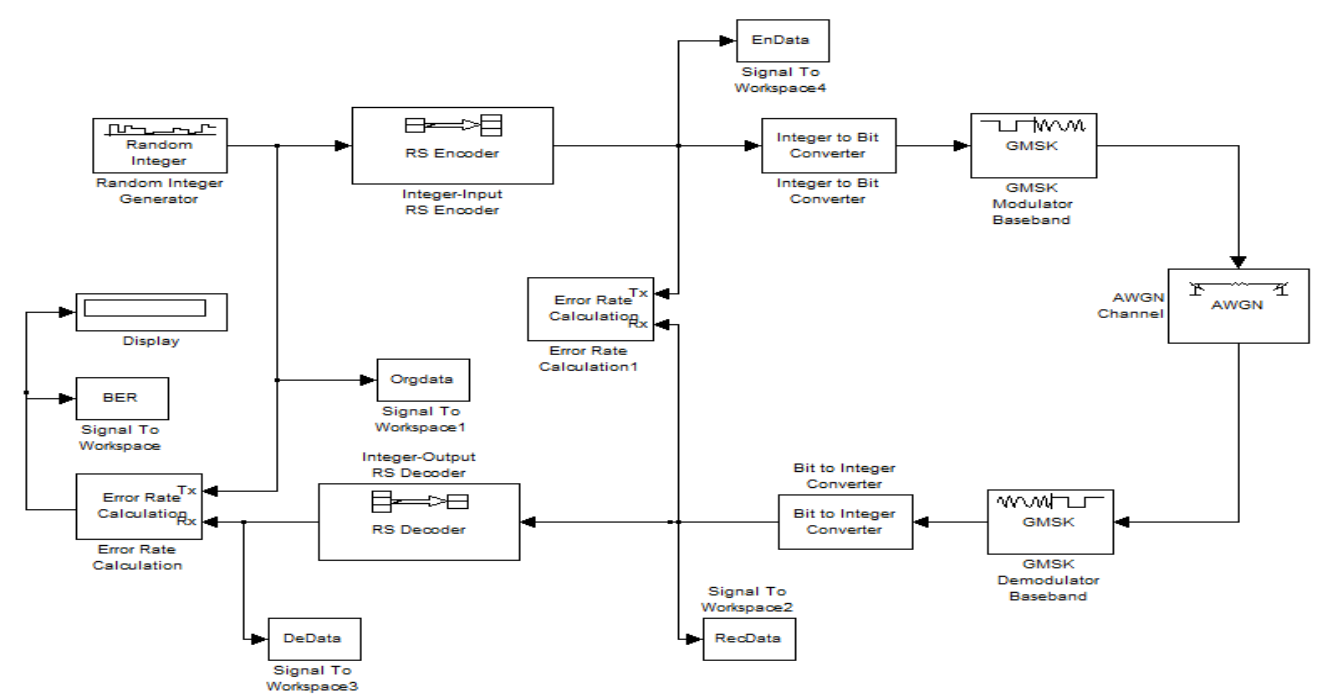

Fig. 3. Proposed Simulation Model

\section{RESULT\& ANALYSIS}

In this section, the parameters used in the simulation model are discussed. Simulation results are also presented. Proposed Simulation is used to evaluate the BER (Bit Error Rates) for different systems. The code parameters used in this simulation areRS $(127, \mathrm{k})$ and $\mathrm{RS}(63, \mathrm{k})$. To ensure the bandwidths of the coded and uncoded systems remain the same, first of all we have to calculate the value of $B_{b} T$. Let us see how to calculate the value of $B_{b} T$, if we set the value of $B_{b} T=X$ for the uncoded system, then $B_{9999} T=Y$ from Figure 2 . When the RS coding rate is $r$, the coded $B_{99: 9} T=Z$ is calculated from $Z=Y \times r$. The corresponding value of $B_{b} T$ is found from Figure 2. The parameters used in the simulations are shown in Table I and Table II.The simulation results are shown in Figures 4-10. From Figure 4, it is clear that as the code rate decreasesor we can say that as the value of $\mathrm{B}_{\mathrm{b}} \mathrm{T}$ decreases, it results in decreasing the bit error probability. When the value of $\mathrm{B}_{\mathrm{b}}$ Treaches to 0.4 for $\mathrm{RS}(63,49)$ codewordprobability of BER reaches a minimum value. but, as the code rate decreases further, probability of BERincreases instead. The reason is that the code rate $r$ decreases but the bit error rate of GMSK $p$ increases, so the net result of decreasing $r$ depends on how much $p$ increases in response to a decrease in $r$. Due to decrease in $r$ when $\mathrm{p}$ increases more, $\mathrm{Pb}$ becomes worse. Similar result can also be find out from Figure 5, 6 and 7 for different system parameters.From Fig 4-10, we can also show the SNR required to achieve $\mathrm{P}_{\mathrm{b}}=10^{-4}$ when the value of $\mathrm{B}_{\mathrm{b}} \mathrm{T}=0.5$ and $\mathrm{B}_{\mathrm{b}} \mathrm{T}=1.0$ for the uncoded system. A smaller value of $\mathrm{B}_{\mathrm{b}} \mathrm{T}$ means a smaller code rate. All the figure shows, as the code rate is decreased, the performance improves until a minimum is reached and then it gets worse. The optimal combination of coding and modulation for a given total transmission bandwidth can be found from these figures. The $(63,49)$ RS shows the maximum gain $(6 \mathrm{~dB})$ achieved over the uncoded system $\left(B_{b} T=0.5\right)$ (see figure 4 and 6 ). For $n=127$, the $(127,107)$ RS has thelargest gain at $(5.8 \mathrm{~dB})$ (see figure 5 and 7 ).

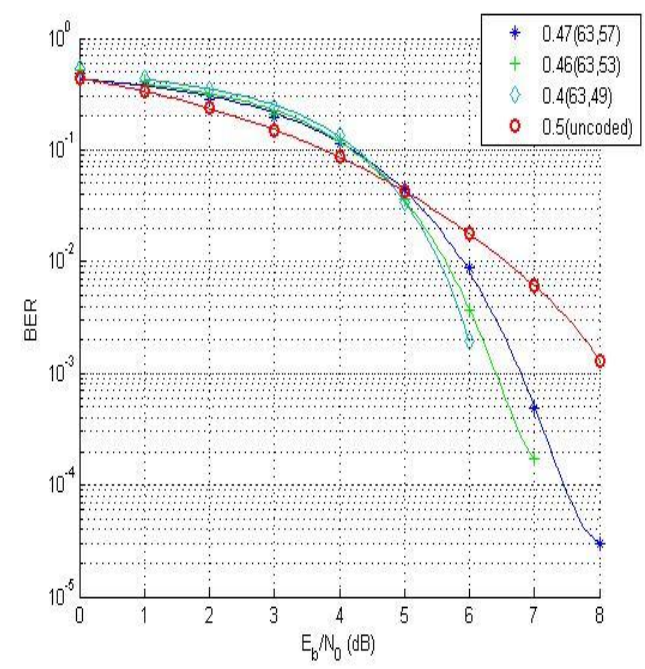

Fig. 4. :Performance for GMSK-RS(63:k) code combinations with the same bandwidth as an uncodedsystemwithB $\mathrm{B}_{\mathrm{b}} \mathrm{T}=0.5$ 


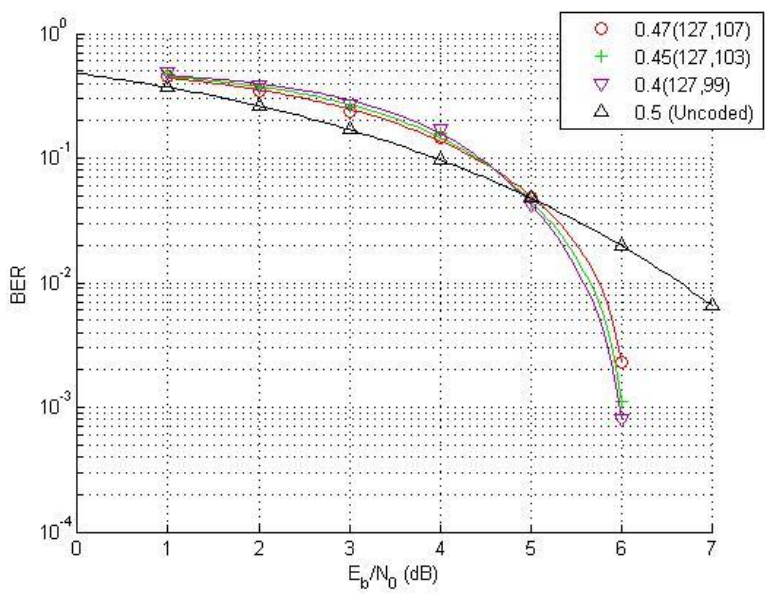

Fig. 5. Performance for GMSK-RS(127;k) code combinations with the same bandwidth as an uncodedsystemwith $\mathrm{B}_{\mathrm{b}} \mathrm{T}=0: 5$

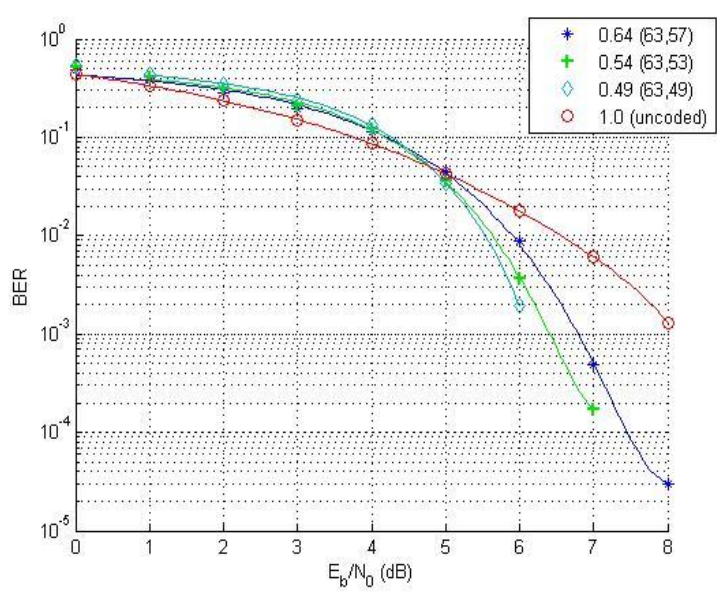

Fig. 6. Performance for GMSK-RS(63;k) code combinations with the same bandwidth as an uncodedsystemwith $\mathrm{B}_{\mathrm{b}} \mathrm{T}=1.0$

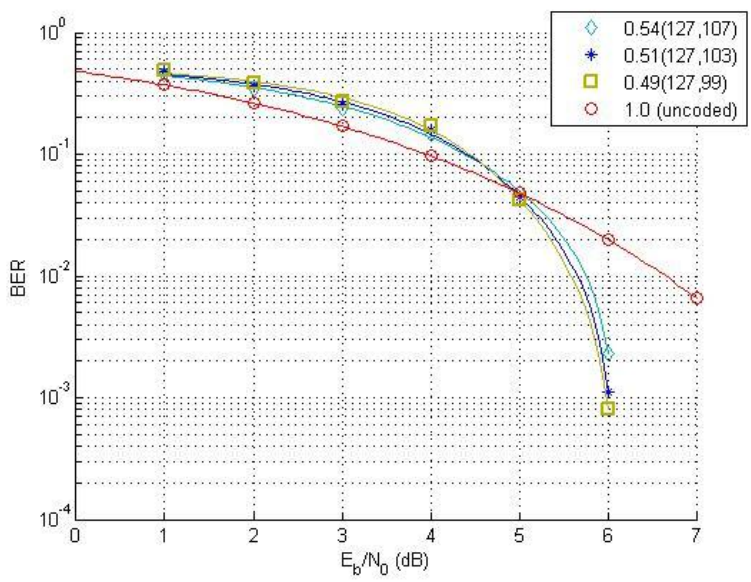

Fig. 7. Performance for GMSK-RS(127:k) code combinations with the same bandwidth as an uncodedsystemwith $\mathrm{B}_{\mathrm{b}} \mathrm{T}=1.0$

Next we performed the simulations for RS codes for different code rates and different block length with fixed number of error correction code. We can see from the figure 8 , as the block length decreases the BER also decreases and the system performance also improves. But the further decrease in block length causes increase in BER (block length $(15,9)$ So here the best result comes out with RS $(31,25)$ with $m=5$ i.e. number of bits per symbol is 5 . We can now say that for RS codes the BER performance improves with the decreasing code rate but by taking large block lengths. The RS code, which is well suited for correction of burst errors, shows a poor BER performance for lower SNR values, because of the random errors introduced by the AWGN. 


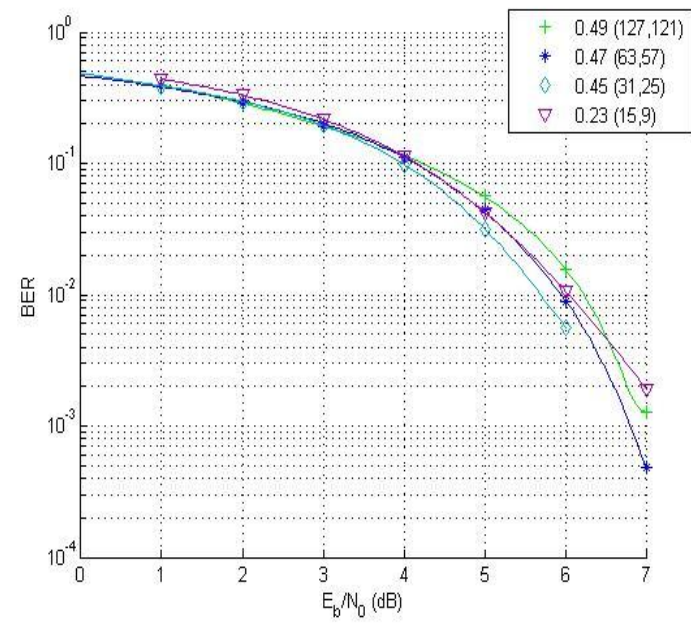

Fig. 8. The BER performance comparison of RS codes for different code rates and different block length but

Fix No. of error correction capabilities

To do this experimentsto be run we use the following RS codes set; RS $(127,123)$, RS $(127,119)$, RS $(127,111)$ and RS $(127,95)$ respectivelyfor simulation results. The above RS code sets have identical codeword symbols (n) whereas the number of datasymbols $(\mathrm{k})$ decreases, which results in decrease in code ratefrom $\mathrm{r}=0.98$ to 0.87 which means increasing in redundancy from 4 to 32 symbols. We have selected the field size 128 , the codeword and data symbols can be measured as 7-bit symbols [8]. From the performance curve given below(see fig 9\&10), it isobserved that as the redundancy increases the error-correcting Reed-Solomon code become more efficient i.e., errorperformance improves as the redundancy increases (lower code rate) as shown in fig. 9.we can also see the similar result from fig. 10.for 63 code word symbols.

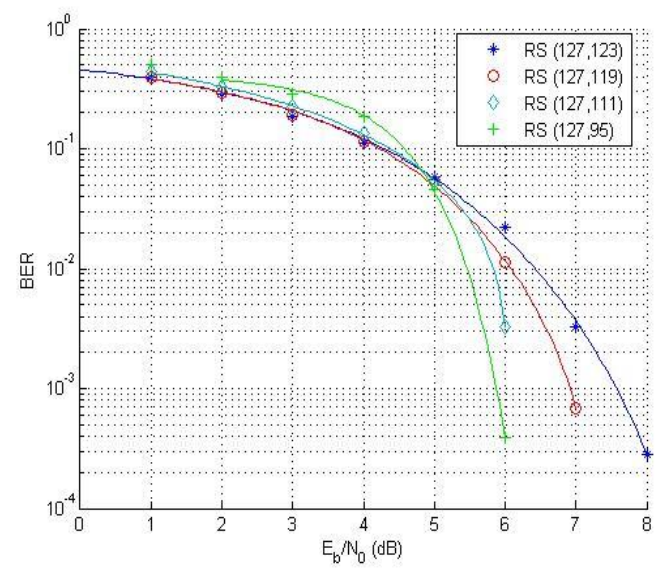

Fig. 9. The BER performance ofReed-Solomon code with increase in Redundancy on codeword 127 having symbol length 7 bits.

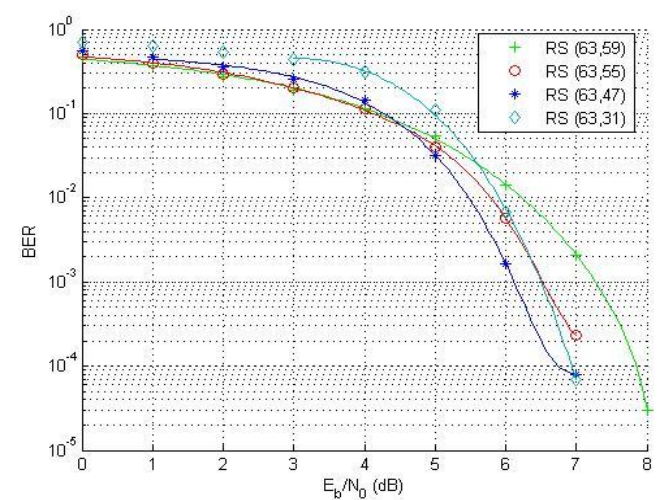

Fig. 10.The BER performance of Reed-Solomon code with increase in Redundancy oncodeword 63 having symbol length 6 bits. 


\section{Conclusion}

In this paper, we examined the performance of communication system overGMSKmodulations which employ RS channel coding. Undera constant bandwidth constraint, we optimized thecombination of coding and modulation.The Proposed system results showthat for same bandwidth as uncoded system, a coded systembit error rate probability performance can be improved for agiven bandwidth by taking smallercoderate. However, when the code rate further decreases, the system performance decreases. For thisreason the optimal code rate was found to be a functionof the total system bandwidth. We also performed the simulations for different code rates and different block length with fixed no of error correction capabilities and result shows that the BER performance can also be improved by decreasing code rate but for large block lengths. In the proposed technique MATLAB simulation is selected as the investigating tool. The results are presented by a plot between the bit error rates (BER) and signal to noise ratio using Monte Carlo simulation.

\section{References}

[1] 12 Wicker, S.B., (1995) "Error Control Systems for Digital Communication and Storage," Prentice-Hall, Upper Saddle River, NJ.

[2] 8. Reed, I. S. and Solomon, G., (1960) "Polynomial Codes Over Certain Finite Fields," SIAM Journal of Applied Math", vol. 8, pp. 300-304.

[3] SAURABH MAHAJAN, GURPADAM SINGH," Reed-Solomon Code Performance for M-ary Modulation over AWGN Channel" International Journal of Engineering Science and Technology (IJEST), Vol. 3 No. 5 May 2011

[4] K. Murota and K. Hirade, "GMSK modulation for digital mobile radio telephony," IEEE Trans. Commun, vol.COM-29, pp.10441050, July 1981.

[5] LiDuanMa,David. K Asano, "Performance of GMSK and Reed-Solomon Code Combinations," The 27th Symposium on Information Theory and Its Applications (SITA2004) Gero, Gifu, Japan, Dec. 14-17, 2004.

[6] I. S. Reed and G. Solomon, "Polynomial Codes over Certain Finite Fields," SI AM Journal of Applied Mathematics, Volume 8, pp. 300-304,1960

[7] Odenwalder, J.P., Error Control Coding Handbook, Linkabit Corporation, San Diego, CA, July 15,1976. 